This document is confidential and is proprietary to the American Chemical Society and its authors. Do not copy or disclose without written permission. If you have received this item in error, notify the sender and delete all copies.

\title{
Oxytetracycline and ciprofloxacin exposure altered the composition of protistan consumers in an agricultural soil
}

\begin{tabular}{|r|l|}
\hline Journal: & Environmental Science \& Technology \\
\hline Manuscript ID & es-2020-02531z.R1 \\
\hline Manuscript Type: & Article \\
\hline Author: & 15 -Jun-2020 \\
\hline Complete List of Authors: & $\begin{array}{l}\text { Nguyen, Bao Anh; University of Melbourne Faculty of Veterinary Science } \\
\text { Parkville Campus, Agricultural and Food Systems } \\
\text { Chen, Qinglin; University of Melbourne Faculty of Veterinary and } \\
\text { Agricultural Sciences, School of Agriculture and Food } \\
\text { He, Jizheng; University of Melbourne Faculty of Veterinary and } \\
\text { Agricultural Sciences } \\
\text { Hu, Hangwei; University of Melbourne, Faculty of Veterinary and } \\
\text { Agricultural Sciences }\end{array}$ \\
\hline
\end{tabular}

\section{SCHOLARONE \\ Manuscripts}


Title page

Oxytetracycline and ciprofloxacin exposure altered the composition of protistan consumers in an agricultural soil

\author{
Bao-Anh T. Nguyen ${ }^{\dagger}$, Qing-Lin Chen ${ }^{\dagger}, *$, Ji-Zheng He ${ }^{\dagger}$, Hang-Wei Hu${ }^{\dagger}, *$ \\ ${ }^{\dagger}$ Faculty of Veterinary and Agricultural Sciences, The University of Melbourne, Parkville VIC \\ 3010, Australia
}




\section{$1 \quad$ ABSTRACT}

2 Protists, an integral component of soil microbiome, are one of the main predators of bacteria.

3 Bacteria can produce toxic secondary metabolites e.g. antibiotics to fight stress under the predation

4 pressure of protists; however, impacts of antibiotics on the profile of protists in soils remain

5 unclear. Here, we constructed a microcosm incubation to investigate the effects of two common

6 antibiotics, oxytetracycline and ciprofloxacin, on the protistan and bacterial communities in arable

7 soils. Rhizaria were the most abundant protist supergroup, followed by Amoebozoa, Stramenopiles

8 and Aveolata. Among trophic functional groups, consumers were predominant within the protistan

9 community. The protistan alpha-diversity was not significantly changed, while the bacterial alpha-

10 diversity was decreased under the pressure of antibiotics. Nevertheless, the antibiotic exposure

11 considerably reduced the relative abundance of protistan lineages in Rhizaria and Amoebozoa,

12 which were the dominant supergroups of protistan consumers, while increased the relative

13 abundance of other consumer and phototrophic protists. Altogether, we provide novel

14 experimental evidence that the bacterivorous consumers, an important functional group of protists,

15 were more sensitive to antibiotics than other functional groups. Our findings have potential

16 implications for the induced alterations of protistan community and their ecological functions

17 under the scenarios of projected increasing global antibiotic usage. 


\section{INTRODUCTION}

Protists, primarily microscopic eukaryotes, form a focal, but often overlooked, part of the soil food webs. ${ }^{1}$ They are highly diverse and ubiquitous in the terrestrial ecosystems $\left(10^{4}-10^{8}\right.$ individuals per gram of soils). ${ }^{2}$ Protists can increase the availability of essential nutrients (e.g. carbon, nitrogen, phosphorus, and silicon) for other organisms and plants via photosynthesis ${ }^{3}$ or forming symbiosis with bacteria in nutrient cycling. ${ }^{4-6}$ More importantly, protists are considered as pivotal regulators on the activities, community structure and evolution of microorganisms mainly via their predation on bacteria and fungi (as main food sources) and other microbial eukaryotes. ${ }^{1,7}$ Under the pressure of predation, bacteria can evolve defense mechanisms to fight against protists, such as by excreting antibiotics (e.g. violacein, 2,4-diacetylphloroglucinol, pyoluteorin, pyrrolnitrin, etc.) as secondary metabolites, ${ }^{8-11}$ which is a fundamental biological driver for the antibiotic production and the development of antimicrobial resistance (AMR) in the natural environment. ${ }^{12}$

The overall global antibiotic consumption is predicted to reach a $200 \%$ increase by 2030 compared to the period of $2000-2015,,^{13}$ with $70 \%$ of the overall antibiotics used for growth promotion and disease control in animal husbandry. ${ }^{14}$ Due to overuse or misuse of antibiotics, the rapid development and proliferation of AMR have become one of the major global health concerns. ${ }^{15}$ The discharge of unmetabolized antibiotics into the environment and their effects on the profile of belowground communities are a great concern. ${ }^{16}$ Majority of previous studies examining the effects of antibiotics on soil microorganisms, however, have focused exclusively on bacterial and fungal communities, ${ }^{17-20}$ but not soil protists, a key component of the soil microbiome. Previous studies reported growth inhibition, cyst formation and/or death of protists under pressures of antibiotics released by selected bacteria, $, 8,{ }^{21}$ but these studies were conducted 
41 with several model species of bacteria and protists under in vitro conditions. Our knowledge of the

42 impacts of antibiotics on the growth and diversity of protists in the complex soil environment

43 remains limited. This knowledge is critical to an improved mechanistic understanding and

44 prediction of the responses of protists, a pivotal component in soil food webs, to increasing global 45 use of antibiotics.

46 Therefore, to address this knowledge gap, we selected two commonly-used antibiotics with

47 different action modes, i.e. oxytetracycline (OTC) and ciprofloxacin (CFC), and investigated their

48 effects on the diversity and community composition of protists in a soil microcosm incubation.

49 Both taxonomic composition and trophic functional traits of the protistan community were

50 examined to provide comprehensive insights into the changes in community structure and

51 ecological function traits of protists under the pressure of antibiotics. Notably, we focused on

52 protist consumers due to the dominance of bacterivorous consumers and their integral regulation

53 on bacteria mainly via predation in soil ecosystems. We also compared the responses of protistan

54 communities and bacterial communities to antibiotics during the incubation. We hypothesized that

55 (1) common antibiotics can significantly alter the diversity and composition of soil protist 56 community, as antibiotics may directly act on protist or indirectly through impacting bacterial

57 communities, the food sources for protists; (2) consumer protists are more likely affected by the 58 antibiotics than other functional groups (e.g. phototrophs and parasites), because they directly feed 59 on bacteria that are susceptible to antibiotic exposures. 


\section{MATERIALS AND METHODS}

\section{Soil sampling}

Soil samples were collected from a long-term research vegetable farm at Clyde (38¹3’02”S, 145'33'24'E), Victoria, Australia in June 2019. The agricultural field is planted with celery and had been fertilized with chicken manure and inorganic fertilizers for more than ten years. The antibiotic residue in the chicken manure was not measured and chicken is not treated with antibiotics as growth promoters in Australia. ${ }^{22}$ This site has a mean annual temperature at $19.4{ }^{\circ} \mathrm{C}$ and a mean annual precipitation at $819 \mathrm{~mm}$. Surface soils $(0-15 \mathrm{~cm})$ were taken by mixing multiple soil cores and transported on ice to the laboratory. Soil samples were ground with a jaw crusher and passed through a $2 \mathrm{~mm}$ sieve to remove roots and stones. The soil was characterized as a loamy sand with a $\mathrm{pH}$ of 7.6 . Soil moisture content was $11.18 \%$ measured by the loss of weight after oven drying at $105{ }^{\circ} \mathrm{C}$ for $24 \mathrm{~h}$. Total carbon and total nitrogen were $2.12 \%$ and $0.24 \%$, respectively, as analyzed using a LECO Trumac CN analyzer (LECO, USA).

\section{Laboratory microcosm incubation}

Two commonly used synthetic antibiotics in livestock production and human therapy, ${ }^{16}$ oxytetracycline (OTC; $\mathrm{C}_{22} \mathrm{H}_{24} \mathrm{~N}_{2} \mathrm{O}_{9} 2 \mathrm{H}_{2} \mathrm{O}$; purity: 90.9\%, CAS Number: 6153-64-6, SigmaAldrich, Australia) and ciprofloxacin $\left(\mathrm{CFC} ; \mathrm{C}_{17} \mathrm{H}_{18} \mathrm{FN}_{3} \mathrm{O}_{3}\right.$; purity: $\geqslant 98 \%$, CAS Number: 8572133-1, Sigma-Aldrich, Australia), were selected for the microcosm incubation. The two antibiotics have distinct action modes, with OTC and CFC functioning as bacteriostatic and bactericidal antibiotics to disrupt protein synthesis and DNA replication of bacterial cells, respectively. ${ }^{23,24}$ The environmental concentrations of OTC and CFC normally range from micrograms ( $\mu \mathrm{g})$ to 
81 milligrams (mg) per $\mathrm{kg}$ soil with a typical level of above $1 \mathrm{mg} \mathrm{kg}^{-1}$ in agricultural soils. ${ }^{18,25,26}$

82 Therefore, we used two environment-relevant concentrations ( $1 \mathrm{mg} \mathrm{kg}^{-1}$ dry soil and $5 \mathrm{mg} \mathrm{kg}^{-1} \mathrm{dry}$ soil) of OTC and CFC in this incubation study.

For soil microcosms, twenty grams of soil (oven dry-weight equivalent) were added into each $250 \mathrm{ml}$ vial. There were five treatments with four replicates (shown in Figure. S1), namely control soils without antibiotics, and soils treated with OTC and CFC solutions to achieve two final concentrations ( $1 \mathrm{mg} \mathrm{kg}^{-1}$ dry soil and $5 \mathrm{mg} \mathrm{kg}^{-1}$ dry soil). Soils were thoroughly mixed and soil moisture content was maintained as the first-day condition (11.18\%) using autoclaved deionized water. The incubation vials were loosely covered to maintain aerobic conditions and incubated in the dark at $25^{\circ} \mathrm{C}$. Autoclaved deionized water was supplemented every three days to maintain the water content. Samples were destructively collected at days 1, 7, 14, 21 and 28, and stored at $-20{ }^{\circ} \mathrm{C}$ before molecular analyses.

\section{Characterizing the community compositions of bacteria and protists}

Soil DNA was extracted from $0.25 \mathrm{~g}$ of soil samples using the Powersoil® DNA Isolation Kit (MoBio Laboratories, Carlsbad, CA, USA) following the manufacturer's instructions. The quantity and purity of extracted DNA were evaluated using the NanoDrop spectrophotometer (ND2000c, NanoDrop Technologies, Wilmington, DE, USA).

To determine the composition and diversity of bacterial and protistan communities in soils, the V4 region of the bacterial 16S rRNA gene and the eukaryotic 18S rRNA gene were amplified with the primer sets $515 \mathrm{~F} / 806 \mathrm{R}^{27}$ and TAReuk454FWD1/TAReukREV3 ${ }^{28}$, respectively. The amplicons were purified, quantified, pooled in equimolar and then sequenced on an Illumina MiSeq PE $300 \times 2$ Sequencer (Majorbio Bio-Pharm Technology Co. Ltd., Shanghai, China). Sequences were demultiplexed and trimmed, and the sequences shorter than $75 \mathrm{bp}$ were removed 
104 from the dataset. To guarantee the quality of downstream analyses, we filtered raw pair-end reads 105 to discard those reads containing three or more ambiguous nucleotides and reads with a low 106 average quality score $(\mathrm{Q}<20)$. The generated high-quality sequences were processed and analyzed 107 using the Quantitative Insights Into Microbial Ecology (QIIME) 1.91 pipeline. ${ }^{29}$ UPARSE was 108 taxonomic assignments. The taxonomic classifications of bacterial OTUs were assigned by 111 blasting against the SILVA database (V_138), ${ }^{31}$ while the Protist Ribosomal Reference (PR2) 112 database $\left(V_{-} 4.5\right)^{32}$ was used to assign taxonomic classification to protists.

\section{Functional assignment for the protistan community}

To estimate trophic functional traits of the protistan community, we assigned protistan taxonomic genera into four main functional groups: consumers, parasites, phototrophs and

116 undetermined. ${ }^{33,}{ }^{34}$ Consumers (or predators) represented a group of protists feeding on a wide 117 range of bacteria and fungi (as their favorite foods) as well as preying on other soil eukaryotes 118 (algae, nematodes and other protists). ${ }^{1}$ Bacterivorous predators that prey on bacteria are the most 119 abundant group of consumers. ${ }^{35}$ Parasites are a group of protists parasitizing on various hosts 120 (animals, plants or other organisms), while phototrophs are protist species obtaining energy via 121 photosynthesis. Unknown taxa of protists were classified as "undetermined". Protistan taxa at the 122 genus level are considered to belong to the same trophic functional group. ${ }^{34}$ We adopted the 123 classifications of Dumack et al. (2019) for protist taxa of the supergroup Rhizaria, ${ }^{36}$ and Adl et al. $124(2019)^{34}$ and Voss et al. (2019) $)^{37}$ for protists in other supergroups (Table S1). 
125

126

127

128

129

130

131

132

133

134

135

136

137

138

139

140

141

142

143

144

145

146

\section{Statistical analyses}

Basic mathematical calculations were performed in Microsoft Excel 2016 (Microsoft, USA). Analysis of variance (ANOVA) and Spearman's correlation analyses were used to analyze the differences in diversity, taxonomic compositions and abundance among different treatments over time in SPSS 25 (IBM, USA). $P$ value $<0.05$ was considered as a significant difference. Principal coordinate analysis (PCoA) based on the Bray-Curtis distance and permutational multivariate analysis of variance (PERMANOVA) with the Adonis function were performed to evaluate the profiles of microbial communities using the 'vegan' package ${ }^{38}$ in R. Response ratios based on the mean relative abundance of protists at the class level were calculated by natural log of the ratio of impacts 39,40 .

\section{RESULTS}

\section{Composition of the eukaryotic community}

Sequencing and quality filtering resulted in a total of 5,881,949 eukaryotic $18 \mathrm{~S}$ rRNA gene sequences across all samples, and these sequences were clustered into 4,614 OTUs. After removal of fungal, metazoan, plant (Streptophyta) and unclassified sequences, 2,439,519 high-quality sequences were clustered into 2,573 protist OTUs (55.77\% of total OTUs) from all samples with an average of 24,395 sequences per sample. Protists were the most dominant eukaryote $(41.47 \%$ of the total eukaryotic sequences), followed by fungi $(25.08 \%)$, metazoa $(20.68 \%)$ and other unknown eukaryotes (11.62\%) (Figure. 1A). Plants, represented by Streptophyta, accounted for approximately $1.15 \%$ of the total sequences.

Rhizaria, mostly comprised of the phylum Cercozoa, were the most abundant supergroup of protists $(33.73 \%$ of the total protist sequences) across all soil samples, followed by other 
147 supergroups including Amoebozoa (28.21\%), Stramenopiles (23.21\%) and Alveolata (7.49\%)

148 (Figure. 1B). Opisthokonta and Archaeplastida had similar relative abundances around 3.01\% and $1493.41 \%$, respectively. Three rare supergroups of soil protists were also detected: Hacrobia (0.69\%), 150 Excavata (0.20\%) and Apusozoa (0.06\%).

(A)

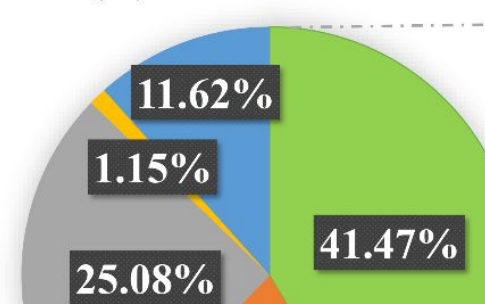

$20.68 \%$
(B)

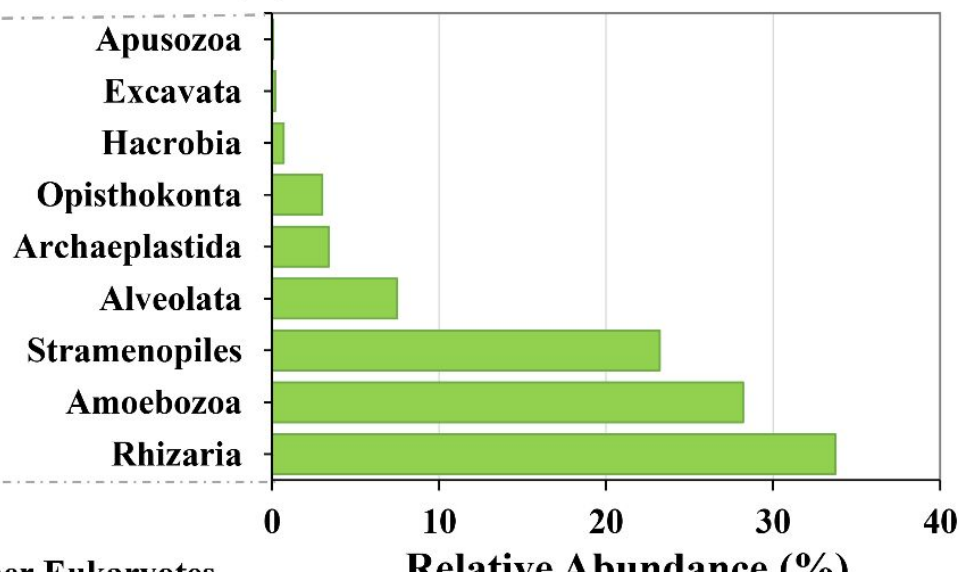

Relative Abundance (\%)
151

152

153

$\square$ Protists $\square$ Metazoa $\square$ Fungi $\square$ Plants $\square$ Other Eukaryotes

Figure 1. The community compositions of eukaryotes across all soil samples (A) and the relative abundance of protist supergroups (B).

\section{Effects of antibiotics on the protistan and bacterial diversity}

The application of OTC or CFC had no significant impacts on the alpha diversity of protists $(\mathrm{F}=0.06, P=0.132)$, but significantly decreased the bacterial diversity $(\mathrm{F}=2.77, P=0.033)$, as revealed by the Shannon index (Figure. S2, Table 1). Incubation time significantly altered the alpha diversity of protists $(\mathrm{F}=5.94, P<0.001)$ (Table 1$)$, which significantly decreased over time during the incubation. Antibiotics, sampling time and their interactions significantly affected the beta diversity of both protists and bacteria, and stronger effects were observed in the diversity of bacterial community $(P$ values $<0.001)$ (Table 1$)$. 
Principal coordinate analysis (PCoA) based on the Bray-Curtis distance differentiated the

163 impacts of OTC and CFC on the protistan community composition at different time points (Figure.

164 2). Significant effects of OTC addition on the community compositions of soil protists were 165 observed at Day 14 (Adonis test, $P<0.05)$ and Day $21(P<0.01$ ), while CFC addition significantly 166 changed the community compositions of soil protists at Day $7(P<0.05)$ and Day $14(P<0.001)$.

167 No significant changes in the profile of protists were observed at the beginning or end of the 168 incubation either for the OTC or CFC treatments $(P>0.05)$.

169 Table 1. Two-way ANOVA analyses to examine the effects of antibiotics, sampling time and their 170 interactive effects on the bacterial and protistan communities.

\begin{tabular}{lcc|cc|cc}
\hline & \multicolumn{2}{c}{ Treatments } & \multicolumn{2}{c}{ Sampling time } & \multicolumn{2}{c}{ Treatment× Sampling time } \\
\cline { 2 - 7 } & $F$ & $P$-values & $F$ & $P$-values & $F$ & $P$-values \\
\hline 1) Bacteria & & & & & & \\
\hline Alpha diversity & $\mathbf{2 . 7 7}$ & 0.033 & $\mathbf{1 4 . 3 8}$ & $<0.001$ & $\mathbf{4 . 5 5}$ & $<0.001$ \\
\hline Beta diversity & $\mathbf{2 2 . 3 7}$ & $<0.001$ & $\mathbf{4 8 . 3 8}$ & $<0.001$ & $\mathbf{2 0 . 3 8}$ & $<0.001$ \\
\hline 2) Protists & & & & & & \\
\hline Alpha diversity & 0.06 & 0.132 & $\mathbf{5 . 9 4}$ & $<0.001$ & 1.62 & 0.08 \\
\hline Beta diversity & $\mathbf{7 . 1 0}$ & $<0.001$ & $\mathbf{2 2 . 0}$ & $<0.001$ & $\mathbf{5 . 9 0}$ & $<0.001$ \\
\hline
\end{tabular}

171 


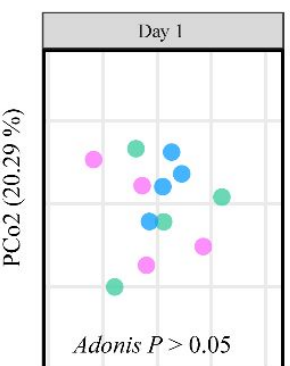

$\mathrm{PCol}(40.28 \%)$

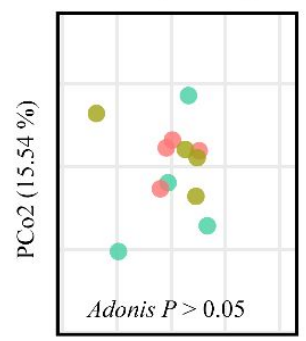

PCo1 $(39.17 \%)$

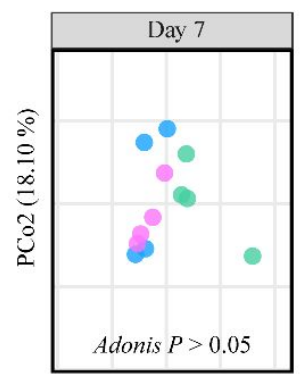

$\mathrm{PCol}(38.28 \%)$

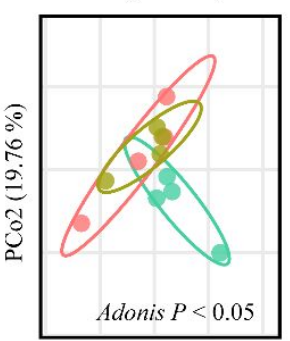

PCol (50.10\%)

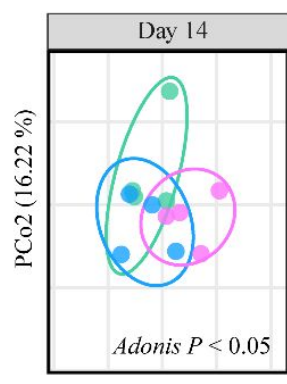

PCo1 $(27.04 \%)$

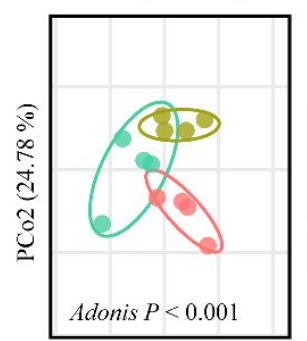

$\mathrm{PCo} 1(35.70 \%)$

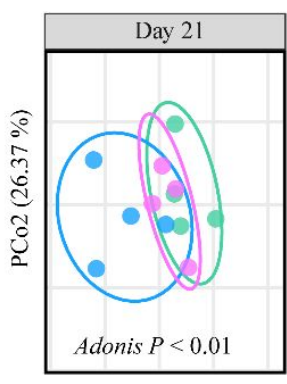

PCo1 $(43.97 \%)$

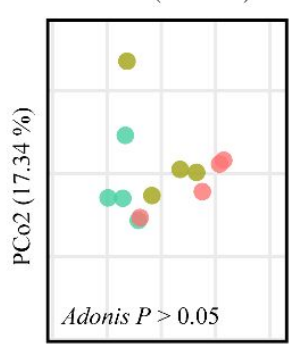

PCol (40.69\%)

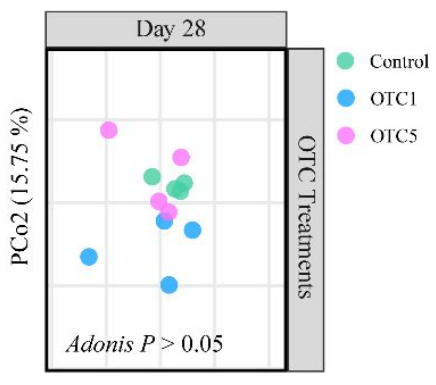

PCol $(43.45 \%)$

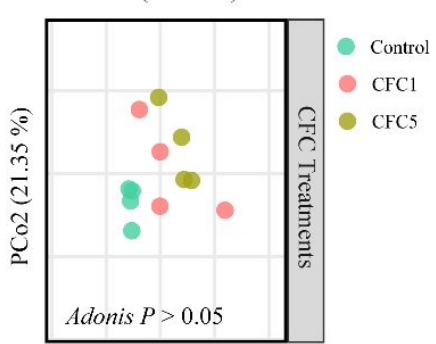

$\operatorname{PCol}(36.32 \%)$

173 Figure 2. Principal coordinate analysis (PCoA) based on the Bray-Curtis distance showing the

174 effects of antibiotics on the changes in the protistan community compositions over time. Adonis test estimates significant differences in the profile of protists among different treatment. OTC, oxytetracycline; CFC, ciprofloxacin.

\section{Effects of antibiotics on the community composition of protists}

The addition of antibiotics had no significant effects on the total relative abundance of protist supergroups $(P>0.05)$, except Archaeplastida (Figures. 3 and S3). However, we identified that some specific protist taxa at the class level in several supergroups including Rhizaria, Amoebozoa, significantly reduced the relative abundance of Endomyxa $(P<0.05)$, while a higher abundance of Filosa-Sarcomonadea was found in all CFC-treated soils $(P<0.05)$.

Among Amoebozoa, most of common amoebozoan lineages at the class level were not affected by antibiotics. However, the relative abundance of a rare taxon Discosea-Flabellinia $(<$ 
187 based response ratios $(P<0.05$, Figure. 4). Likewise, in Stramenopiles, CFC addition had 188 significant effects on less abundant taxa, with an increase of Labyrinthulea in the CFC1 and CFC5 189 treatments and a decline of Bacillariophyta in the CFC5 treatment $(P<0.05$; Figure. 3$)$. Notably, 190 Archaeplastida, entirely composed of green algae Chlorophyta, was the only supergroup being 191 significantly increased by antibiotic addition, with more abundant Chlorophyceae and 192 Trebouxiophyceae at the class level observed in the OTC and CFC1 treatments compared to 193 control soils (Figures. 3 and 4). Similarly, Apusomonadidae_Group-2 of a rare supergroup 194 Apusozoa considerably increased their abundance in the CFC5 treatment $(P<0.01)$. 


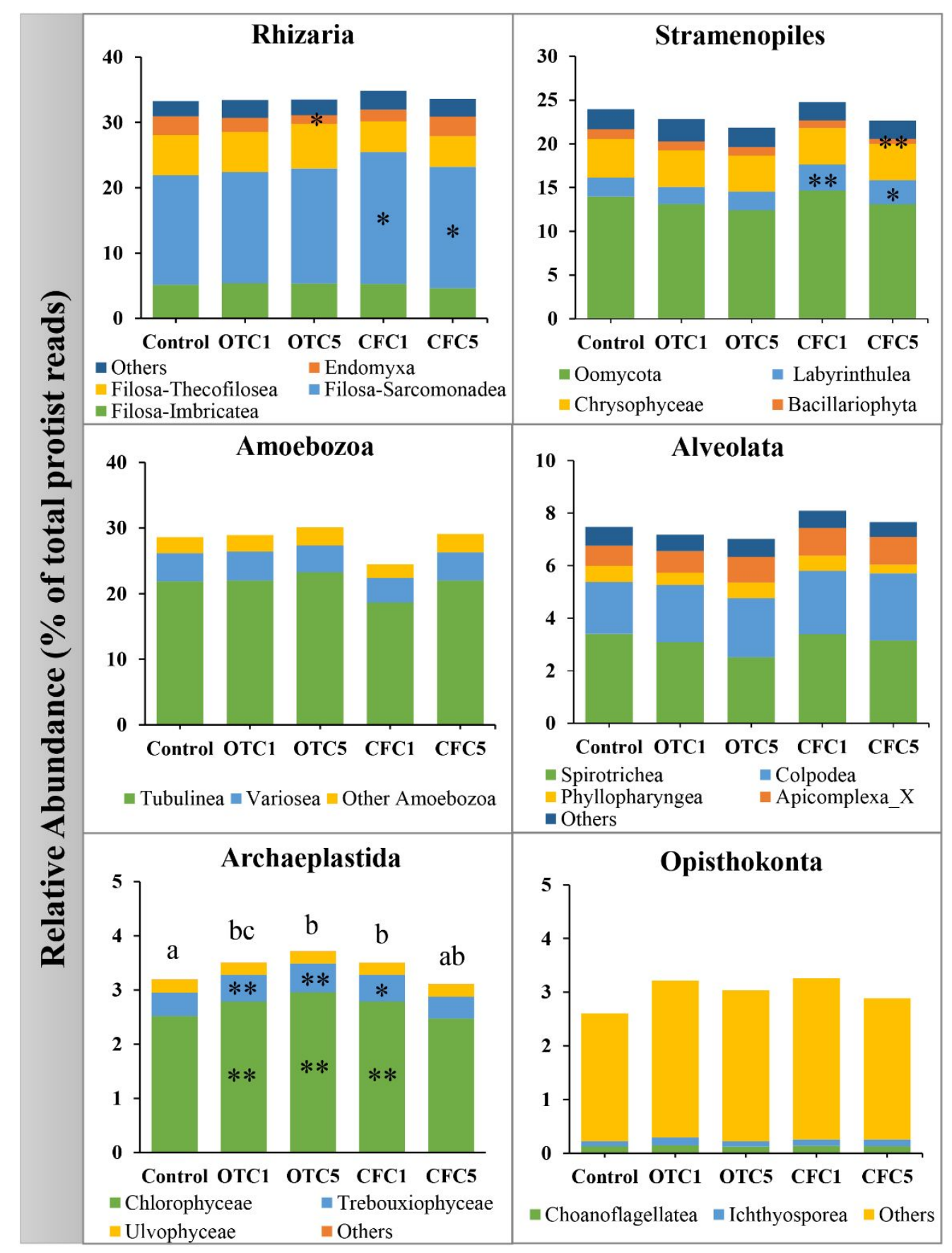

196 Figure 3. Taxonomic profiles of soil protists at the class level for all dominant supergroups.

197 Asterisks or letters indicate significant difference in protistan supergroups in treated samples 198 compared to controls under exposure to oxytetracycline (OTC) and ciprofloxacin (CFC) 199 (ANOVA, $* P<0.05$ and $* * P<0.01)$. 


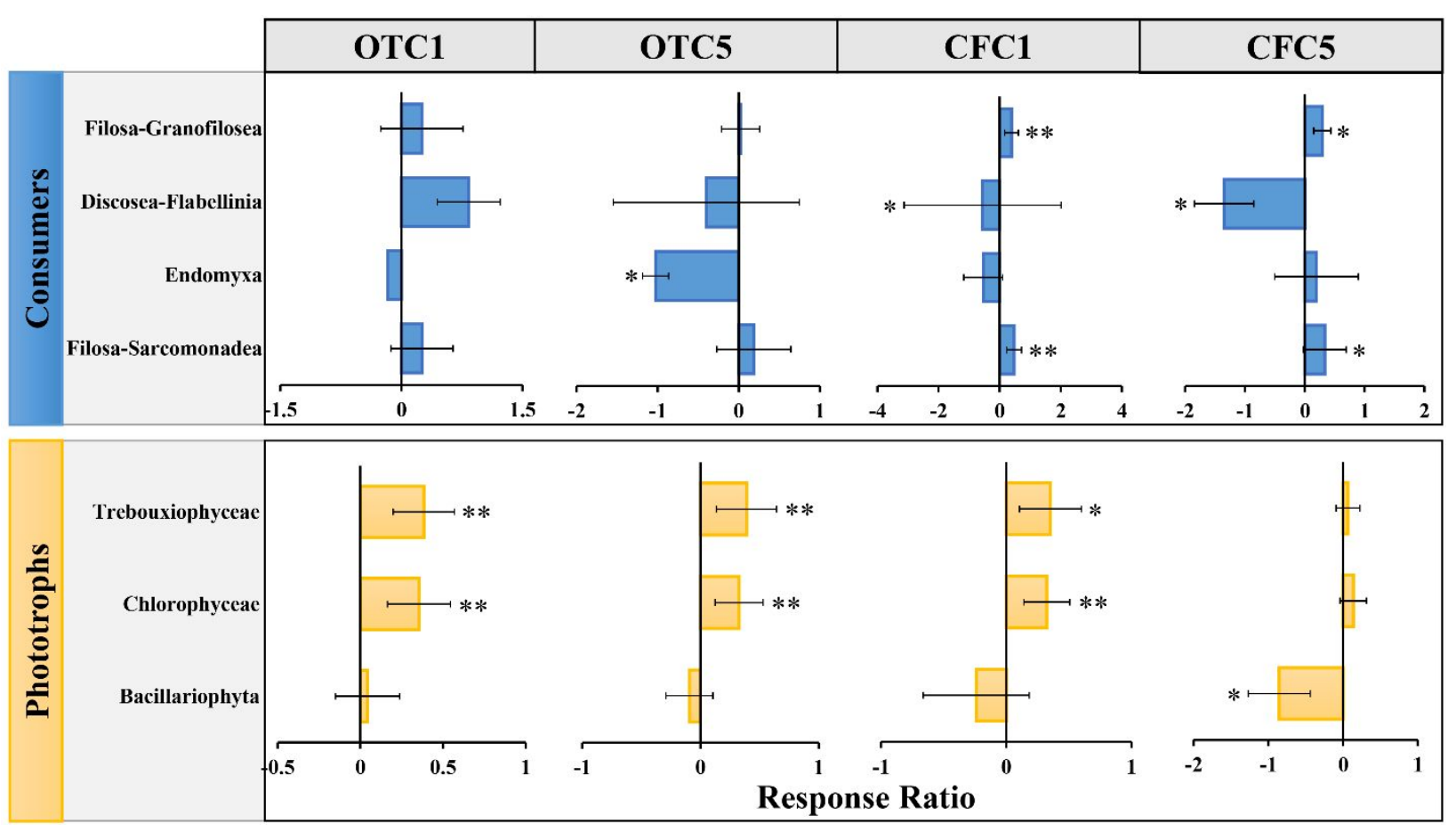

Figure 4. Response ratios of the relative abundances of functional protist lineages to the addition with significant differences compared to controls $(* P<0.05$ and $* * P<0.01$, ANOVA) are plotted on a $\log$ base 2 scale. Error bars indicate standard errors $(n=4)$. OTC, oxytetracycline; CFC, ciprofloxacin.

\section{Effects of antibiotics on functional groups of protists}

Functional groups of protists encompassed consumers (70.08\% of protists), parasites (11.75\%), phototrophs (5.19\%) and undetermined (12.98\%) (Figure. 5A). The composition of

210 (28.26\%), Alveolata (7.20\%) and Stramenopiles (1.93\%) (Figure. 5B). Within consumers, a

211 significant decrease in the relative abundance of Endomyxa (Rhizaria) and Discosea-Flabellinia

212 (Amoebozoa) at the class level was observed in the OTC5 and CFC treatments, respectively 213 (Figure. 4, ANOVA, $P<0.05$ ). In contrast, consumers Filosa-Granofilosea and Filosa- 
214 Sarcomonadea (Rhizaria) were more abundant in the CFC-treated soils than control (Figures. 4 215 and 5C). Parasites and undetermined groups showed a similar pattern, with no significant effects 216 of antibiotics in their relative abundances observed among treatments $(P>0.05)$ (Figure. S4). In 217 addition, the relative abundance of phototrophic protists was significantly increased in the OTC1 218 treatment $(P<0.05)$ (Figure. S4). Among them, the relative abundance of Chlorophyceae and 219 Trebouxiophyceae (Archaeplastida) increased in the OTC and CFC1 treatments, while the relative 220 community over time (Figure. S5). The OTC addition only changed the community composition of protistan consumers at Day 14 (Adonis test, $P<0.01$ ), and the consumer community 224 composition was significantly affected by CFC at Day $7(P<0.05)$ and Day $14(P<0.01)$.
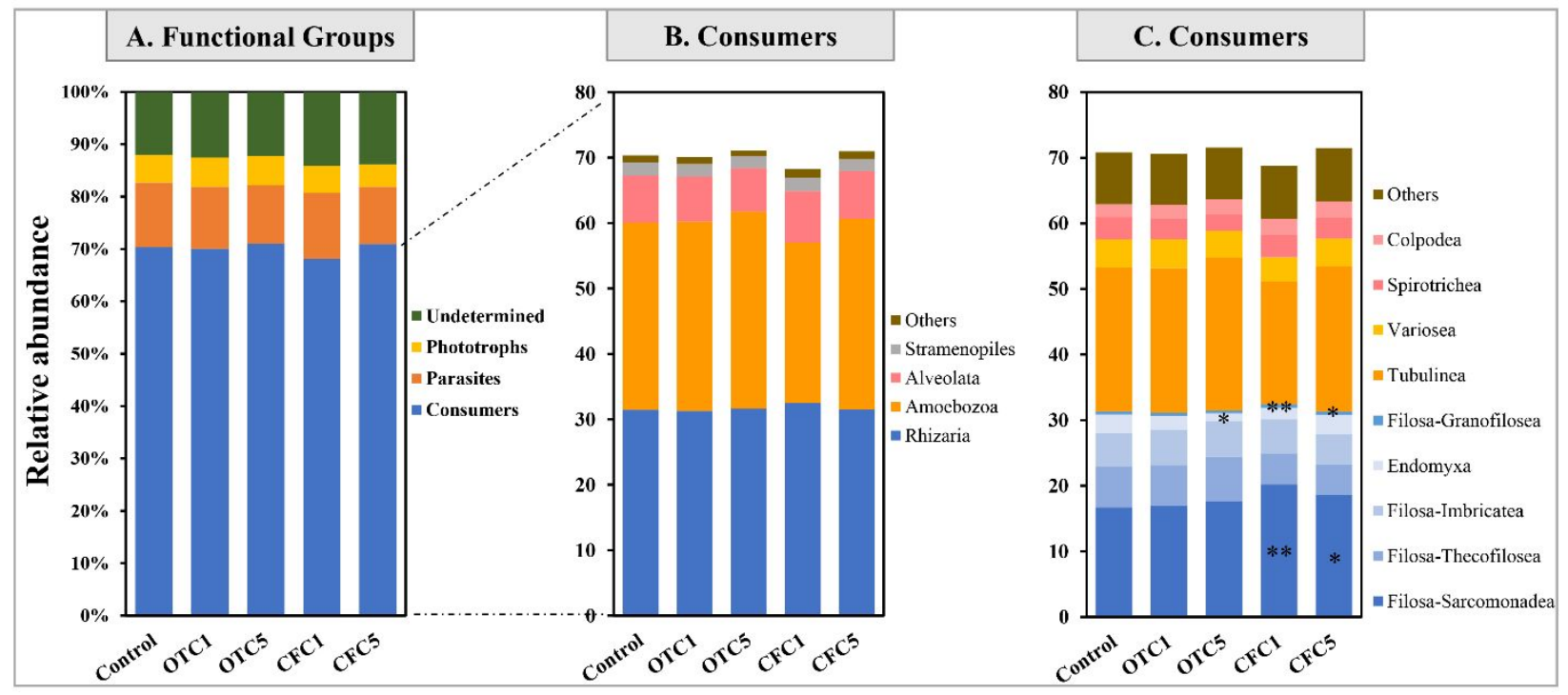

Figure 5. The relative abundances of functional groups of protists (A), and major protistan consumers at the supergroup level (B) and the class level (C) across the five treatments. Significant

228 differences compared to controls under exposure to oxytetracycline (OTC) and ciprofloxacin 229 (CFC) are indicated by asterisks: ${ }^{*} P<0.05$ and $* * P<0.01$ (ANOVA, LSD test). 


\section{DISCUSSION}

In our study, in contrast to the hypothesis 1 , the addition of antibiotics did not change the alpha diversity of soil protists but significantly decreased the bacterial diversity (Table 1). Antibiotics imposed a weaker pressure on the protistan diversity than the bacterial diversity, which indicated that protists may have a higher tolerance to antibiotic perturbation or only a small proportion of protistan taxa are sensitive to antibiotics. Nevertheless, the antibiotics caused significant changes in the community composition of soil protists and consumers during the middle of the incubation but no difference among treatments observed at the end of the incubation (Figures 2 and S5), which could be possibly explained by (i) the rapid degradation of antibiotics in soil microcosms; (ii) the recovery of soil protists at the late stage under the reduced pressure of antibiotics; and (iii) the recovery of bacterial biomass at the late stage under the reduced antibiotic pressure, resulting in more food sources for protistan consumers. Within the trophic functional groups of protists, we also found that the relative abundance of some protist lineages of the consumers was substantially influenced by the antibiotic disturbance (Figure 4). Protistan consumers primarily function as bacterivores (feeding on bacteria), eukaryvores (feeding on algae, fungi, protists and other eukaryotes) and omnivores (feeding on eukaryotes and bacteria). ${ }^{41,42}$ In agreement with the hypothesis 2 , protistan consumers were more affected by antibiotics than other functional groups of protists. The reduction in the abundance of bacterivorous consumers Discosea-Flabellinia (Amoebozoa) were found in response to CFC, whereas the higher concentration of OTC5 caused a negative influence on the abundance of the Endomyxa. In contrast, the relative abundance of bacterivorous consumers Filosa-Granofilosea and FilosaSarcomonadea increased in the CFC-treated soils. Probably, under the antibiotic pressure, Filosa- 
252 Sarcomonadea and Filosa-Granofilosea might take advantage of antibiotic-susceptible bacteria as

253 food source to maintain and promote their growth.

In this microcosm incubation, the different responses of protistan consumers to antibiotic

255 exposure could be due to direct and indirect effects of antibiotics. Firstly, we proposed that the 256 negative influence of antibiotics on protistan consumers might be directly caused by toxicity of 257 antibiotics which adversely impact the cellular processes of protist population (including growth 258 restriction, encystation, cyst reactivation, paralysis and cell lysis $)^{9-11}$ and consequently result in the 259 fatality of sensitive protists. This is supported by the insignificant correlations between the relative 260 abundance of protistan consumers (Endomyxa and Discosea-Flabellinia) and the bacterial taxa 261 (showing significant changes in their abundances during incubation) under the OTC and CFC 262 exposure (Table S2). These results indicate that the decreasing abundance of the bacterivorous 263 consumers Endomyxa and Discosea-Flabellinia might be due to the direct toxicity of antibiotics 264 OTC and CFC, rather than the alteration of their food sources (bacteria). In particular, the 265 bactericide CFC imposed a stronger stress on the protistan community than the bacteriostatic OTC, 266 and more significant impacts were rendered by the higher concentrations $\left(5 \mathrm{mg} \mathrm{kg}^{-1}\right)$ of both 267 antibiotics (Figures. 3, 4, 5C and S3). The bactericidal CFC might be more toxic to the protistan 268 community than bacteriostatic OTC, probably owing to their different action modes: CFC is a 269 bactericide to cause cellular death by targeting DNA replication, while OTC functions as a 270 bacteriostat with inhibitory effects on protein synthesis. ${ }^{24,43}$ However, molecular mechanisms of 271 antibiotic actions on protists need to be deciphered in future studies. Secondly, antibiotics could 272 have indirect effects on the protistan community by impacting their main preys - bacteria. 273 Antibiotics are renowned as a strong selection pressure for the development and evolution of the 274 antibiotic resistance of bacterial community, ${ }^{44}$ and thus the food source of some protist consumers 
275 may be considerably shifted by the increasing population of antibiotic resistant bacteria (ARB)

276 and death of the susceptible bacterial population. In this work, the OTC and CFC treatments

277 dramatically altered the relative abundances of some bacterial taxa (e.g., Rokubacteria,

278 Latescibacteria and Hydrogenedentes), which had significantly negative associations with the

279 increasing abundance of the protistan consumers Filosa-Granofilosea and Filosa-Sarcomonadea

280 (Table S2). The antibiotic pressures might increase the availability of antibiotic-susceptible

281 bacteria and non-antibiotic-producing ARB, which could be a food source to maintain and

282 facilitate the growth and development of the protistan consumers. Therefore, it is not surprising to 283 observe the decrease in some protistan taxa but increase in other taxa of consumers under the 284 pressure of antibiotics in our incubation study. Together, our results highlight that consumers, an 285 important functional group of protists that mainly prey on bacteria, are susceptible to antibiotics 286 in the complex soil matrix, and further support the previous perceptions that predation-resistant 287 bacteria can produce antibiotics to fight against the protist predation in natural environments.

We found the increasing abundance of the phototrophic group and two phototrophic taxa 289 Trebouxiophyceae and Chlorophyceae (Archaeplastida, Chlorophyta), in opposition to an 290 insignificant difference in parasitic protists during the incubation with antibiotics (Figures. 4 and 291 S4). The protistan consumers including eukaryvores and omnivores can prey on phototrophic 292 protists (e.g., algae). The addition of antibiotics in the soil microcosms might impose a pressure 293 on the growth of antibiotic-susceptible bacteria, and these susceptible bacteria might be a major 294 energy source for soil protist consumers. Hence, there might probably be less predatory activities 295 of consumers on algae and other phototrophic protists, and this could maintain and increase the 296 abundance of phototrophic groups and taxa. The well-adaptation and viability of phototrophic 297 protists were also reported after the addition of fertilizers ${ }^{45}$ and in a severe desert condition ${ }^{46}$. For 
298 parasites, the stability of this trophic group in our investigation may be explained by that action 299 modes of antibiotics do not target on main hosts (soil metazoa) of parasitic protists while parasites

300

301

302

303

304

305

306

307

308

309

310

311

312

313

314

315

316 317 the incubation with OTC and CFC.

318 AUTHOR INFORMATION

319 Corresponding Authors

320

\section{ASSOCIATED CONTENT}

\section{Supporting Information}

are mainly associated and driven by their hosts (plants and other soil organisms). ${ }^{47}$

In conclusion, we provide novel evidence that short-term addition of common synthetic antibiotics significantly influenced the community composition of soil protists and the relative abundances of specific protist lineages in the laboratory microcosm incubation. Our results highlight that consumers, an important functional group of protists that mainly prey on bacteria, are sensitive to both of the antibiotics. Our findings support the previous perceptions that bacteria can produce antibiotics to fight against the protist predation in natural environments, and further caution the potentially substantial impacts of synthetic antibiotics on the protistan community and their ecological functions under the scenarios of projected increasing global antibiotic usage.

The experimental design of the laboratory microcosm study; alpha diversity of the protist and bacterial communities; changes in the relative abundance of protistan taxa at the class level in rare protist supergroups; the relative abundances of functional groups of protists at the supergroup and class levels; the effects of antibiotics on the distribution patterns of protistan consumers; taxonomic classifications and functional trait assignments of protists; significant correlations (Spearman) between the abundance of bacterial and consumer taxa with significant changes within and after

\footnotetext{
Hang-Wei Hu, Email: hang-wei.hu@unimelb.edu.au;
} 
321 Qing-Lin Chen, Email: qinglin.chen@unimelb.edu.au

322 ORCID

323 Bao-Anh Thi Nguyen: 0000-0001-6185-2790

324 Qing-Lin Chen: 0000-0002-5648-277X

325 Ji-Zheng He: 0000-0002-9169-8058

326 Hang-Wei Hu: 0000-0002-3294-102X

327 Notes

328 The authors declare no competing financial interest.

329 ACKNOWLEDGMENTS

330 This work was financially supported by the Australian Research Council (DP170103628). 


\section{REFERENCES}

1. Geisen, S., Mitchell, E. A., Adl, S., Bonkowski, M., Dunthorn, M., Ekelund, F., Fernández, L. D., Jousset, A., Krashevska, V., and Singer, D. (2018) Soil protists: a fertile frontier in soil biology research, FEMS Microbiol. Rev. 42, 293-323.

2. Adl, S. M., and Coleman, D. C. (2005) Dynamics of soil protozoa using a direct count method, Biol. Fertil. Soils 42, 168-171.

3. Jassey, V. E., Signarbieux, C., Hättenschwiler, S., Bragazza, L., Buttler, A., Delarue, F., Fournier, B., Gilbert, D., Laggoun-Défarge, F., and Lara, E. (2015) An unexpected role for mixotrophs in the response of peatland carbon cycling to climate warming, Sci. Rep. 5,16931 .

4. Gast, R. J., Sanders, R. W., and Caron, D. A. (2009) Ecological strategies of protists and their symbiotic relationships with prokaryotic microbes, Trends Microbiol. 17, 563-569.

5. Creevy, A. L., Fisher, J., Puppe, D., and Wilkinson, D. M. (2016) Protist diversity on a nature reserve in NW England-With particular reference to their role in soil biogenic silicon pools, Pedobiologia 59, 51-59.

6. Trap, J., Bonkowski, M., Plassard, C., Villenave, C., and Blanchart, E. (2016) Ecological importance of soil bacterivores for ecosystem functions, Plant Soil 398, 1-24.

7. Dumack, K., Pundt, J., and Bonkowski, M. (2019) Food choice experiments indicate selective fungivorous predation in fisculla terrestris (Thecofilosea, Cercozoa), J. Eukaryot. Microbiol. 66, 525-527.

8. Jousset, A., and Bonkowski, M. (2010) The model predator Acanthamoeba castellanii induces the production of 2, 4, DAPG by the biocontrol strain Pseudomonas fluorescens Q2-87, Soil Biol. Biochem. 42, 1647-1649. 
354 9. Jousset, A., Lara, E., Wall, L. G., and Valverde, C. (2006) Secondary metabolites help

355 biocontrol strain Pseudomonas fluorescens CHA0 to escape protozoan grazing, Appl. Environ. Microbiol. 72, 7083-7090.

10. Matz, C., Deines, P., Boenigk, J., Arndt, H., Eberl, L., Kjelleberg, S., and Jürgens, K. (2004) Impact of violacein-producing bacteria on survival and feeding of bacterivorous nanoflagellates, Appl. Environ. Microbiol. 70, 1593-1599.

11. Mazzola, M., De Bruijn, I., Cohen, M. F., and Raaijmakers, J. M. (2009) Protozoan-induced regulation of cyclic lipopeptide biosynthesis is an effective predation defense mechanism for Pseudomonas fluorescens, Appl. Environ. Microbiol. 75, 6804-6811.

12. Nguyen, B.-A. T., Chen, Q.-L., He, J.-Z., and Hu, H.-W. (2019) Microbial regulation of natural antibiotic resistance: Understanding the protist-bacteria interactions for evolution of soil resistome, Sci. Total Environ., 135882.

13. Klein, E. Y., Van Boeckel, T. P., Martinez, E. M., Pant, S., Gandra, S., Levin, S. A., Goossens, H., and Laxminarayan, R. (2018) Global increase and geographic convergence in antibiotic consumption between 2000 and 2015, Proc. Natl. Acad. Sci. U.S.A. 115, E3463-E3470.

14. Jechalke, S., Heuer, H., Siemens, J., Amelung, W., and Smalla, K. (2014) Fate and effects of veterinary antibiotics in soil, Trends Microbiol. 22, 536-545.

15. Hernando-Amado, S., Coque, T. M., Baquero, F., and Martínez, J. L. (2019) Defining and combating antibiotic resistance from One Health and Global Health perspectives, Nat. Microbiol. 4, 1432-1442. 
375 16. Cycoń, M., Mrozik, A., and Piotrowska-Seget, Z. (2019) Antibiotics in the soil

376

377

378

379

380

381

382

383

384

385

386

387

388

389

390

391

392

393

environment—-degradation and their impact on microbial activity and diversity, Front. Microbiol. 10, 338-338.

17. Ollivier, J., Kleineidam, K., Reichel, R., Thiele-Bruhn, S., Kotzerke, A., Kindler, R., Wilke, B.-M., and Schloter, M. (2010) Effect of sulfadiazine-contaminated pig manure on the abundances of genes and transcripts involved in nitrogen transformation in the rootrhizosphere complexes of maize and clover, Appl. Environ. Microbiol. 76, 7903-7909.

18. Cui, H., Wang, S.-P., Fu, J., Zhou, Z.-Q., Zhang, N., and Guo, L. (2014) Influence of ciprofloxacin on microbial community structure and function in soils, Biol. Fertil. Soils $50,939-947$.

19. Nazir, R., Shen, J.-P., Wang, J.-T., Hu, H.-W., and He, J.-Z. (2017) Fungal networks serve as novel ecological routes for enrichment and dissemination of antibiotic resistance genes as exhibited by microcosm experiments, Sci. Rep. 7, 1-13.

20. Orlewska, K., Markowicz, A., Piotrowska-Seget, Z., Smoleń-Dzirba, J., and Cycoń, M. (2018) Functional diversity of soil microbial communities in response to the application of cefuroxime and/or antibiotic-resistant Pseudomonas putida strain MC1, Sustainability 10,3549 .

21. Song, C., Mazzola, M., Cheng, X., Oetjen, J., Alexandrov, T., Dorrestein, P., Watrous, J., Van Der Voort, M., and Raaijmakers, J. M. (2015) Molecular and chemical dialogues in bacteria-protozoa interactions, Sci. Rep. 5, 12837.

22. Hu, H. W., Han, X. M., Shi, X. Z., Wang, J. T., Han, L. L., Chen, D., and He, J. Z. (2016) Temporal changes of antibiotic-resistance genes and bacterial communities in two contrasting soils treated with cattle manure, FEMS Microbiol. Ecol. 92(2). 
23. Brown, D. (2015) Antibiotic resistance breakers: can repurposed drugs fill the antibiotic discovery void?, Nat. Rev. Drug Discov. 14, 821-832.

24. Lázár, V., Nagy, I., Spohn, R., Csörgő, B., Györkei, Á., Nyerges, Á., Horváth, B., Vörös, A., Busa-Fekete, R., and Hrtyan, M. (2014) Genome-wide analysis captures the determinants of the antibiotic cross-resistance interaction network, Nat. Commun. 5, 4352.

25. Zhao, L., Dong, Y. H., and Wang, H. (2010) Residues of veterinary antibiotics in manures from feedlot livestock in eight provinces of China, Sci. Total Environ. 408, 1069-1075.

26. Widyasari-Mehta, A., Hartung, S., and Kreuzig, R. (2016) From the application of antibiotics to antibiotic residues in liquid manures and digestates: a screening study in one European center of conventional pig husbandry, J. Environ. Manage. 177, 129-137.

27. Bates, S. T., Berg-Lyons, D., Caporaso, J. G., Walters, W. A., Knight, R., and Fierer, N. (2011) Examining the global distribution of dominant archaeal populations in soil, ISME J. $5,908$.

28. Stoeck, T., Bass, D., Nebel, M., Christen, R., Jones, M. D., BREINER, H. W., and Richards, T. A. (2010) Multiple marker parallel tag environmental DNA sequencing reveals a highly complex eukaryotic community in marine anoxic water, Mol. Ecol. 19, 21-31.

29. Caporaso, J. G., Kuczynski, J., Stombaugh, J., Bittinger, K., Bushman, F. D., Costello, E. K., Fierer, N., Pena, A. G., Goodrich, J. K., and Gordon, J. I. (2010) QIIME allows analysis of high-throughput community sequencing data, Nat. Methods 7, 335.

30. Edgar, R. C. (2013) UPARSE: highly accurate OTU sequences from microbial amplicon reads, Nat. Methods 10, 996. 
419 31. Quast, C., Pruesse, E., Yilmaz, P., Gerken, J., Schweer, T., Yarza, P., Peplies, J., and

420

421

422

423

424

425

426

427

428

429

430

431

432

433

434

435

436

437

438

439

440

441

Glöckner, F. O. (2012) The SILVA ribosomal RNA gene database project: improved data processing and web-based tools, Nucleic Acids Res. 41, D590-D596.

32. Guillou, L., Bachar, D., Audic, S., Bass, D., Berney, C., Bittner, L., Boutte, C., Burgaud, G., de Vargas, C., and Decelle, J. (2012) The Protist Ribosomal Reference database (PR2): a catalog of unicellular eukaryote small sub-unit rRNA sequences with curated taxonomy, Nucleic Acids Res. 41, D597-D604.

33. Oliverio, A. M., Geisen, S., Delgado-Baquerizo, M., Maestre, F. T., Turner, B. L., and Fierer, N. (2020) The global-scale distributions of soil protists and their contributions to belowground systems, Sci. Adv. 6, eaax 8787.

34. Adl, S. M., Bass, D., Lane, C. E., Lukě̌, J., Schoch, C. L., Smirnov, A., Agatha, S., Berney, C., Brown, M. W., and Burki, F. (2019) Revisions to the classification, nomenclature, and diversity of eukaryotes, J. Eukaryot. Microbiol. 66, 4-119.

35. Hiltunen, T., Friman, V.-P., Kaitala, V., Mappes, J., and Laakso, J. (2012) Predation and resource fluctuations drive eco-evolutionary dynamics of a bacterial community, Acta oecologica $38,77-83$.

36. Dumack, K., Fiore-Donno, A. M., Bass, D., and Bonkowski, M. (2020) Making sense of environmental sequencing data: Ecologically important functional traits of the protistan groups Cercozoa and Endomyxa (Rhizaria), Mol. Ecol. Resour. 20, 398-403.

37. Voss, C., Fiore-Donno, A. M., Guerreiro, M. A., Peršoh, D., and Bonkowski, M. (2019) Metatranscriptomics reveals unsuspected protistan diversity in leaf litter across temperate beech forests, with Amoebozoa the dominating lineage, FEMS Microbiol. Ecol. 95, fiz142. 
442 38. Oksanen, J., Kindt, R., Legendre, P., O’Hara, B., Stevens, M. H. H., Oksanen, M. J., and 443 Suggests, M. (2007) The vegan package, Community ecology package 10, 631-637.

444 39. Hedges, L. V., Gurevitch, J., and Curtis, P. S. (1999) The meta-analysis of response ratios in 445 experimental ecology, Ecology 80, 1150-1156.

446 40. Clark, M., and Tilman, D. (2017) Comparative analysis of environmental impacts of 447 agricultural production systems, agricultural input efficiency, and food choice, Environ. Res. Lett. 12, 064016.

449 450 451 452 453 454 455 456 457 458 459 460 461 462

41. Dumack, K., Fiore-Donno, A. M., Bass, D., and Bonkowski, M. (2019) Making sense of environmental sequencing data: Ecologically important functional traits of the protistan groups Cercozoa and Endomyxa (Rhizaria), Mol. Ecol. Resour.

42. Fiore-Donno, A. M., Richter-Heitmann, T., Degrune, F., Dumack, K., Regan, K. M., Marhan, S., Boeddinghaus, R., Rillig, M. C., Friedrich, M. W., and Kandeler, E. (2019) Functional traits and spatio-temporal structure of a major group of soil protists (Rhizaria: Cercozoa) in a temperate grassland, Front. Microbiol. 10, 1332.

43. Ocampo, P. S., Lázár, V., Papp, B., Arnoldini, M., Zur Wiesch, P. A., Busa-Fekete, R., Fekete, G., Pál, C., Ackermann, M., and Bonhoeffer, S. (2014) Antagonism between bacteriostatic and bactericidal antibiotics is prevalent, Antimicrob. Agents Chemother. 58, 4573-4582.

44. Cycoń, M., Mrozik, A., and Piotrowska-Seget, Z. (2019) Antibiotics in the Soil Environment—Degradation and Their Impact on Microbial Activity and Diversity, Front. Microbiol. 10. 
463 45. Gilbert, D., Amblard, C., Bourdier, G., and Francez, A.-J. (1998) The microbial loop at the 464 surface of a peatland: structure, function, and impact of nutrient input, Mol. Ecol. 35, 83465 93.

466

46. Lewis, L. A., and Lewis, P. O. (2005) Unearthing the molecular phylodiversity of desert soil green algae (Chlorophyta), Syst. Biol. 54, 936-947.

468

47. Schulz, G., Schneider, D., Brinkmann, N., Edy, N., Daniel, R., Polle, A., Scheu, S., and Krashevska, V. (2019) Changes in trophic groups of protists with conversion of rainforest into rubber and oil palm plantations, Front. Microbiol. 10, 240. 

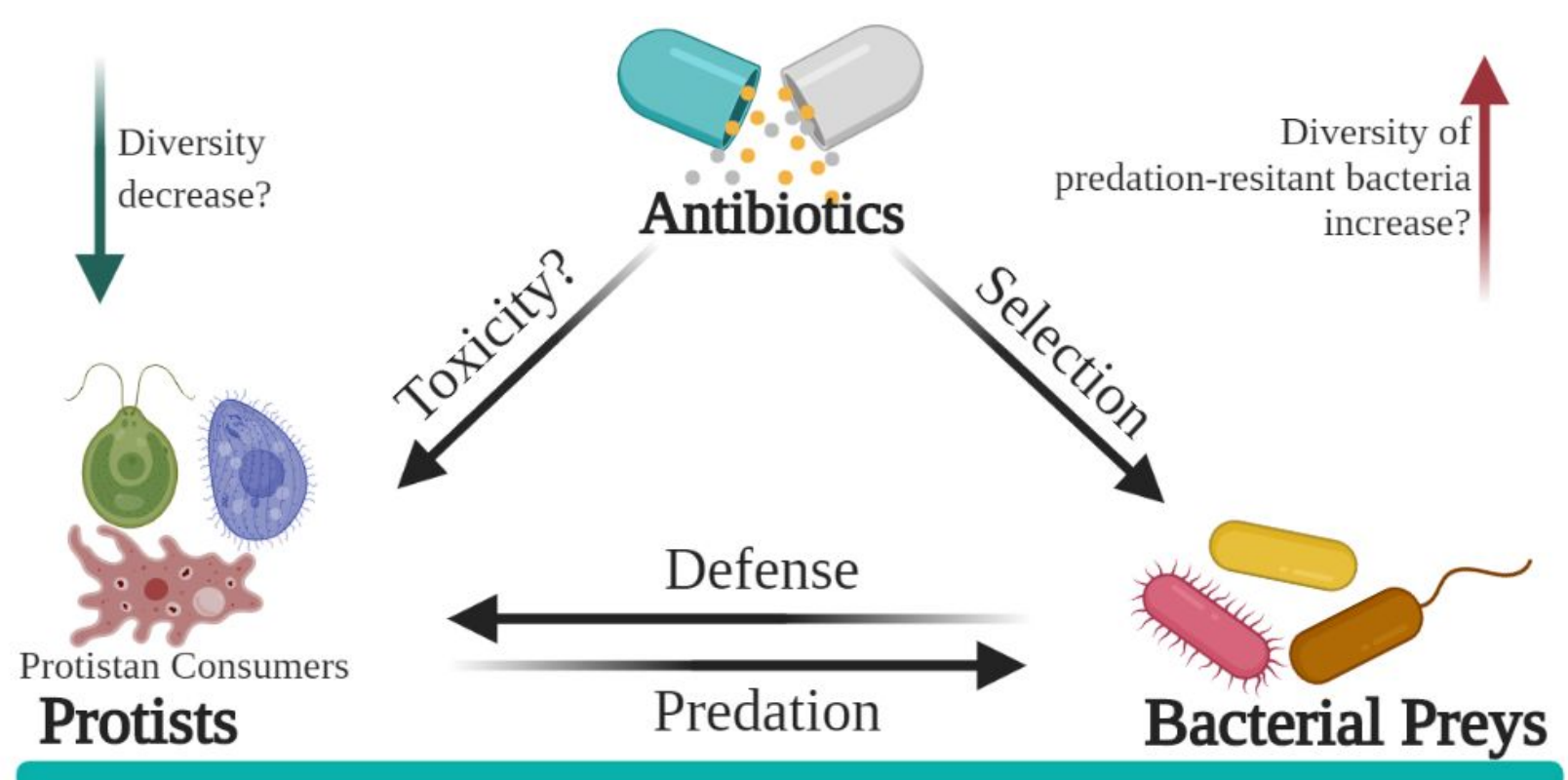

473

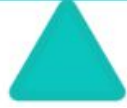




\section{University Library}

\section{- M M N E R VA A gateway to Melbourne's research publications}

Minerva Access is the Institutional Repository of The University of Melbourne

Author/s:

Nguyen, B-AT;Chen, Q-L;He, J-Z;Hu, H-W

Title:

Oxytetracycline and Ciprofloxacin Exposure Altered the Composition of Protistan Consumers in an Agricultural Soil

Date:

2020-08-04

Citation:

Nguyen, B. -A. T., Chen, Q. -L., He, J. -Z. \& Hu, H. -W. (2020). Oxytetracycline and Ciprofloxacin Exposure Altered the Composition of Protistan Consumers in an Agricultural Soil. ENVIRONMENTAL SCIENCE \& TECHNOLOGY, 54 (15), pp.9556-9563. https:// doi.org/10.1021/acs.est.0c02531.

Persistent Link:

http://hdl.handle.net/11343/291501 